\title{
Hif-2alpha mediates UV-induced apoptosis through a novel ATF3-dependent death pathway
}



In this study, we describe a novel activating transcription factor 3 (ATF3)-dependent death pathway triggered by ultraviolet (UV) irradiation. We demonstrate that ATF3 contributes to UV-induced apoptosis through the regulation of hypoxia inducible factor (Hif)-2alpha expression, which in turn induces the expression of proapoptotic genes, such as Caspase7 or TRAIL (tumor necrosis factor (ligand) superfamily, member 10). Gain of function of Hif-2alpha as well as ATF3 is sufficient to trigger cell death, whereas loss of function of both proteins drastically inhibits UV-induced apoptosis. Repression of Hif-2alpha strongly impairs ATF3-mediated death, providing evidences that Hif-2alpha is the major death effector of ATF3. In addition, Hif-1alpha, already known as a proapoptotic gene, upon UV irradiation, is not able to compensate for the lack of Hif-2alpha expression, thereby confirming the major contribution of Hif-2alpha in UV-mediated cell death. We further demonstrate that this cascade of gene activation depends on p38 and c-Jun N-terminal kinase (JNK) activity. Impairment of such a pathway is likely to contribute to oncogenesis by promoting survival of cells that could accumulate severe chromosomal alterations.

Cell Death and Differentiation (2008) 15, 1472-1480; doi:10.1038/cdd.2008.74; published online 30 May 2008

\begin{abstract}
Oncogenesis and tumor progression involve the accumulation of mutations and genomic alterations. Environmental genotoxic stress, such as ultraviolet (UV) light, is one of the major causes of genomic instability. During such a stress, normal cells orientate their physiological response toward DNA repair or, if the extent of damage is too severe, undergo apoptosis. This process, deleterious for cell survival, is crucial because it leads to the destruction of cells that bear the risk of becoming tumorigenic. Such an antitumor mechanism acts through coordinated regulation at different levels (transcriptional and post-transcriptional), which leads to the precise expression of genes involved in the control of proliferation, DNA repair and apoptosis. ${ }^{1,2}$ Thus, upon chronic genotoxic stress, putative cancer cells undergo a selective pressure to modulate their gene regulation during the DNA damage response to ensure survival while accumulating genomic mutations. This leads to clonal expansion of damaged cells, progression into the precancerous state and, likely, to uncontrolled proliferation., Therefore, identifying the genes and pathways required for genotoxic stress response will provide important information on the onset of carcinogenesis as well as on the rational approach to potential drug design.

We have recently reported that alteration of the expression of early growth response (Egr)-1 during UV-mediated genotoxic stress has serious consequences for DNA damage and proapoptotic gene expression leading to cell survival., 5 Among other early response transcription factors, activating transcription factor 3 (ATF3) is greatly induced following UV irradiation. ${ }^{7,8}$ ATF 3 is a member of the ATF/CREB subfamily
\end{abstract}

of the basic-region leucine zipper (bZIP) family, which includes members of the CCAAT/enhancer-binding protein and the AP-1 families. ${ }^{9,10}$ Members of the ATF/CREB family bind to a consensus DNA sequence (TGACGTCA) ${ }^{11}$ and may activate or repress gene transcription. ATF3 protein is expressed at low levels in normal and quiescent cells but may be strongly induced in a variety of tissues by different stress signals like ischemia, wounding, homocysteine, UV, ionizing radiation and genotoxic chemical agents. ${ }^{12-14}$ Several pathways are responsible for ATF3 induction, such as ATM and Nibrin 1, c-Jun N-terminal kinase (JNK) and $\mathrm{NF}-\mathrm{kB}$ in a p53-dependent or independent manner. ${ }^{8,12,14}$ Interestingly, induction of ATF3 often correlates with cellular damages, suggesting an important role during stress response. ${ }^{9,15}$ However, its function is not clearly understood and both detrimental and protective effects have been reported. ${ }^{14,16,17}$ Despite the fact that, upon UV-mediated genotoxic stress, ATF3 induction occurs in a P53-independent manner, ${ }^{12}$ neither its regulation nor its mechanism of action is clearly understood.

In this report, our aim is to characterize the function of ATF3 and its mechanism of action during a UV-mediated genotoxic stress. We show that $p 38$ and JNK activation are necessary for ATF3 induction in a transcriptional manner. We demonstrate that ATF3 is responsible for the expression of hypoxia inducible factor (Hif)-2alpha, which in turn contributes to the expression of TRAIL (tumor necrosis factor (ligand) superfamily, member 10) upon UV irradiation. Moreover, ATF3 as well as Hif-2alpha knockdown drastically reduces

\footnotetext{
${ }_{1}^{1}$ INSERM U898, Nice F-06107, France; ${ }^{2}$ Université de Nice-Sophia Antipolis, Nice F-06107, France; ${ }^{3}$ Institute of Signaling, Developmental Biology and Cancer Research, CNRS UMR 6543, University of Nice, Centre A. Lacassagne, 33 Avenue Valombrose, Nice 06189, France; ${ }^{4}$ INSERM U576, Nice F-06202, France and ${ }^{5}$ Department of Biochemical Genetics, Medical Research Institute, Tokyo Medical and Dental University, Yushima, 1-5-45 Tokyo, Japan

${ }^{*}$ Corresponding author: T Virolle, INSERM U898, Faculté de Médecine, 28 Avenue Valombrose, 06107 Nice cedex 2, France.

Tel: + 04933776 20; Fax: + 049381 14 04; E-mail: virolle@ @unice.fr

Keywords: UV; Hif-2alpha; apoptosis; ATF3; TRAIL

Abbreviations: ATF3, activating transcription factor 3; UV, ultraviolet; Hif-2alpha, hypoxia inducible factor 2 alpha; Hif-1alpha, hypoxia inducible factor 1 alpha; TRAIL, tumor necrosis factor (ligand) superfamily (member 10); JNK, c-Jun N-terminal kinase; EGR1, early growth response 1; EGR2, early growth response 2

Received 29.10.07; revised 10.4.08; accepted 11.4.08; Edited by JP Medema; published online 30.5.08
} 
a
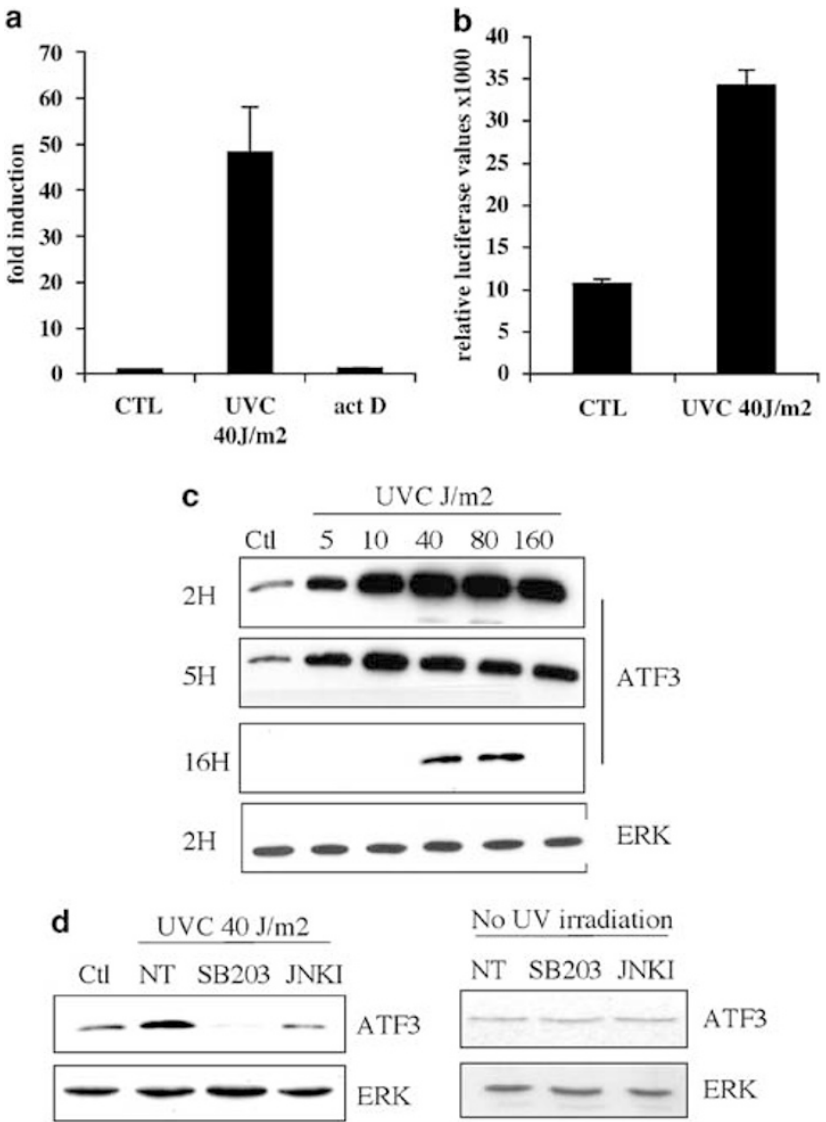

No UV irradiation

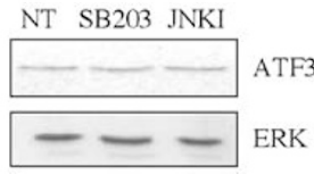

Figure 1 UV treatment induces ATF3 expression. (a) Total RNA was prepared from HaCaT cells exposed to UVC $\left(40 \mathrm{~J} / \mathrm{m}^{2}\right)$ in the presence or not of actinomycin D. ATF3 mRNA expression was assessed by one-step real-time RT-PCR as described in Materials and Methods. CTL corresponds to the non-irradiated condition. The fold inductions correspond to the ratio between control (CTL) and irradiated cells. Error bars are the S.D. of three separate experiments in duplicate. (b) Luciferase ATF3 promoter construct was transfected and cloned into HeLa cells treated or not by UVC $\left(40 \mathrm{~J} / \mathrm{m}^{2}\right)$. CTL corresponds to the non-irradiated condition. Error bars are the S.D. of three separate experiments in quadruplicate. (c) Nuclear extracts were prepared from $\mathrm{HaCaT}$ cells according to a time course following increasing amount of UV irradiation $\left(5-160 \mathrm{~J} / \mathrm{m}^{2}\right)$. Western blot analysis was then performed with antibodies against ATF3 and ERK as loading control. Ctl corresponds to non-irradiated condition. (d) P38 and JNK are responsible for ATF3 induction. ATF3 protein expression was analyzed in UV $\left(40 \mathrm{~J} / \mathrm{m}^{2}\right)$-irradiated $\mathrm{HaCaT}$ cells. When indicated, the cells were treated with P38 or JNK inhibitors (SB203 and JNK1, respectively) $1 \mathrm{~h}$ prior to stimulation. NT corresponds to UVCirradiated, non-treated condition (left panel) and to non-treated condition only (right panel). Ctl corresponds to non-irradiated, non-treated condition. Western blot was performed as described in Materials and Methods

UV-mediated apoptosis, whereas their overexpression is sufficient to promote cell death. Therefore, our results identify a novel pathway, gatekeeper of tumorigenesis, which contributes to the orchestration of apoptosis of damaged cells.

\section{Results}

UV mediates ATF3 transcriptional expression in human epidermal cells. To test ATF3 expression in the context of UV-irradiated epidermal cells, we assessed ATF3 mRNA expression by real-time PCR in UV-irradiated HaCaT cells.
Although ATF3 gene expression was almost undetectable under control conditions, it was rapidly and strongly induced by UVC irradiation (Figure 1a). Incubation of the cells with actinomycin $D$ prior to stimulation led to the inhibition of ATF3 mRNA expression (Figure 1a), suggesting that ATF3 induction, at least at early stages of the genotoxic stress response, was strictly dependent on a transcriptional event. Accordingly, ATF3 promoter activity was significantly enhanced in transiently transfected HeLa cells (Figure 1b). To further investigate at the protein level, $\mathrm{HaCaT}$ cells were irradiated with $5-160 \mathrm{~J} / \mathrm{m}^{2}$ UVC. Nuclear proteins were extracted 2, 5 and $16 \mathrm{~h}$ following treatment. As seen in Figure 1c, significant ATF3 protein expression peaked at $2 \mathrm{~h}$ and started to decrease at $5 \mathrm{~h}$, becoming almost undetectable at $16 \mathrm{~h}$. Optimal induction was observed at 40 and $80 \mathrm{~J} / \mathrm{m}^{2}$ UVC. Taken together, these results showed that ATF3 gene is transcriptionally induced in epidermal cells in response to UV stress, resulting in high levels of protein production at early stages. To identify the molecular pathway involved in ATF3 induction, HaCaT cells were preincubated with LY, Wortmannin, U0126, SB203 and JNK inhibitors (which inhibit PI3kinase, MEK, P38 and JNK, $1 \mathrm{~h}$ before genotoxic stress treatment. UV irradiation led to a strong stimulation of ATF3 protein expression, which was drastically reduced when $\mathrm{P} 38$ and JNK activities were blocked (Figure 1d). No inhibitory effect was observed with LY, Wortmannin and U0126 (Supplementary data Figure 1A). The induction of ATF3 promoter activity by UV was also lost when the stress kinases were inhibited (Supplementary data Figure 1B). This demonstrates that the activities of P38 and JNK are necessary for UV-mediated ATF3 induction through a transcriptional event. Similar results were observed in HeLa cells.

ATF3 is a mediator of UV-induced apoptosis. A recent study reported that ATF3 mediates UV-induced cell death and alters proliferation in fibroblasts. ${ }^{18}$ To determine whether ATF3 knockdown could also affect epithelial cell survival following UV stress, we used a previously described ${ }^{19}$ siRNA (siATF3(1)) to specifically inhibit its expression. As illustrated in Figure 2a, siATF3(1) efficiently inhibited ATF3 protein expression in $\mathrm{HaCaT}$ and HeLa cells. HeLa and HaCaT were irradiated with a single low dose of $40 \mathrm{~J} / \mathrm{m}^{2}$ UVC and apoptosis was monitored $24 \mathrm{~h}$ later by measuring caspase $3 /$ 7 activity. In the context of HeLa and HaCaT cells, inhibition of ATF3 reduced caspase $3 / 7$ activity (Figure $2 b$ and $c$ ) leading to a strong increase in the survival rate as assessed by trypan blue staining (Figure $2 e$ ), whereas no effect was observed on cell viability in non-irradiated epithelial cells (data not shown). Similar results were obtained when ATF3 knockdown was performed with another sequence of siRNA (siATF3(2)) (Figure 2e). The same experiment performed on irradiated WT and ATF3-/-MEFs further confirmed the requirement of ATF3 expression in UV-mediated cell death (Figure 2d). Interestingly, overexpression of ATF3 protein (Figure $3 a$ ) led to a significant reduction in the number of living cells (Figure 3b), which was correlated with a significant increase in caspase $3 / 7$ activity (Figure 3c), thereby demonstrating a direct role of ATF3 in apoptosis. Taken together, these results indicate that expression of 
a
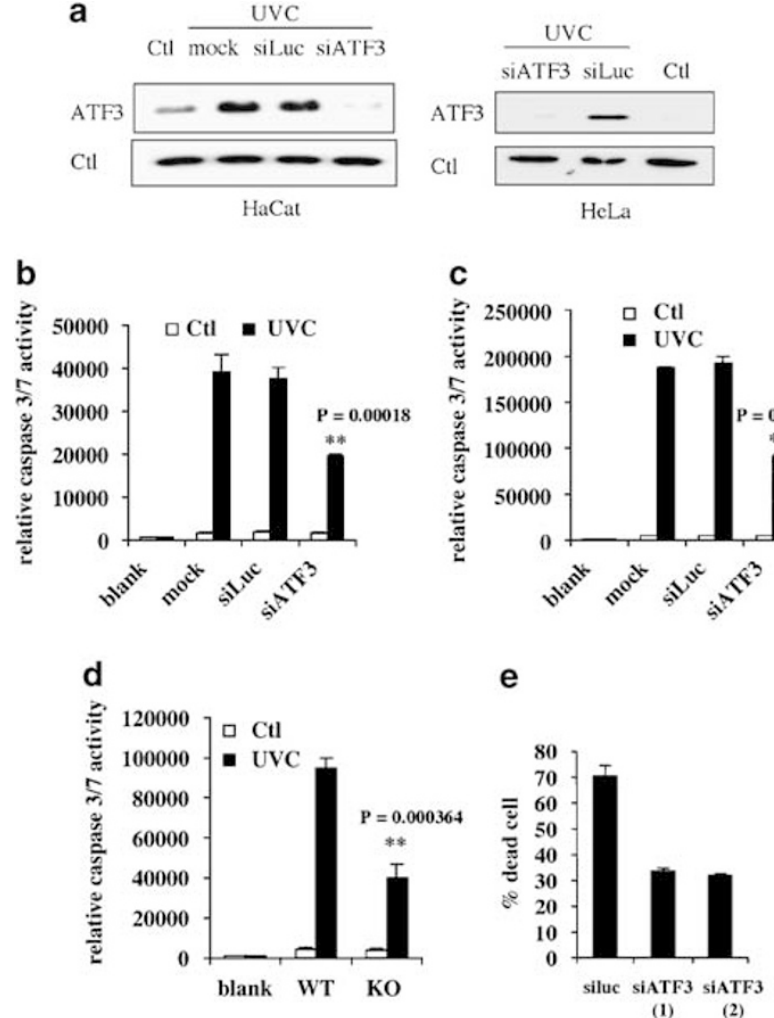

Figure 2 ATF3 expression is required for UV-mediated cell death. (a) siATF3(1) efficiently repressed ATF3 protein expression. $\mathrm{HaCaT}$ cells were transfected with siATF3 and siLuc siRNA as a control. After $24 \mathrm{~h}$, the cells were UV irradiated $\left(40 \mathrm{~J} / \mathrm{m}^{2}\right)$ or not $(\mathrm{Ctl}) .2 \mathrm{~h}$ later, the cells were lysed and the samples were analyzed by western blotting with antibodies to ATF3. (b-d) ATF3-deficient cells are less sensitive to death than ATF3-expressing cells. siATF3- or siLuc-transfected HeLa (b) HaCaT (c) or ATF3 KO and WT MEF (d) cells were UVC-irradiated $\left(40 \mathrm{~J} / \mathrm{m}^{2}\right)$. Apoptosis was quantified by caspase $3 / 7$ assay, $24 \mathrm{~h}$ after UVC irradiation. The figures represent three separate experiments. Statistical analysis was performed (Student's $t$-test): ${ }^{*} P$-value that represents a significant difference between siLucand siATF3-treated cells, WT and ATF3 KO cells. (e) HeLa cells were transfected with siATF3(1), siATF3(2), or siLuc as control. After 24h, the cells were UV irradiated $\left(40 \mathrm{~J} / \mathrm{m}^{2}\right)$ and the percentage of dead cell has been measured by trypan blue staining

ATF3 is sufficient to mediate cell death and strongly contributes to the induction of an optimal apoptotic process in response to UV irradiation.

Hif-2alpha expression depends on ATF3. To determine the effectors through which ATF3 can promote UV-induced apoptosis, we compared the transcriptome of HeLa cells deficient in ATF3 expression (siATF3) with that of control siLuc-transfected cells, $2 \mathrm{~h}$ and $30 \mathrm{~min}$ following UVC $\left(40 \mathrm{~J} / \mathrm{m}^{2}\right)$ irradiation. Total RNA was extracted and hybridized on a human pangenomic microarray. ${ }^{20}$ Among the genes that were upregulated by ATF3 (i.e., downregulated by knockdown of ATF3), we found Hif-1 and Hif-2alpha. Although Hif-1alpha transcripts were only slightly affected, a strong repression of Hif-2alpha transcripts was induced by the knockdown of ATF3 (Figure 4a). Accordingly, ATF3 expression upon UV irradiation occurred earlier than Hif2alpha mRNA induction, as seen by the assessment of time-course expressions (Figure 4b). Inhibition of ATF3

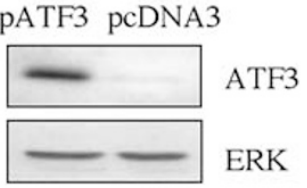

b

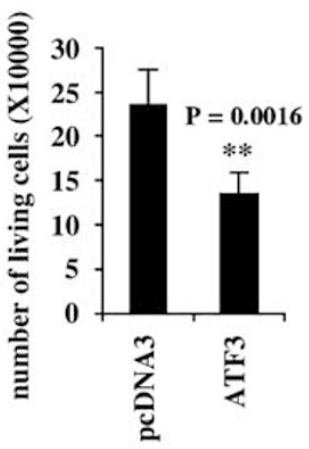

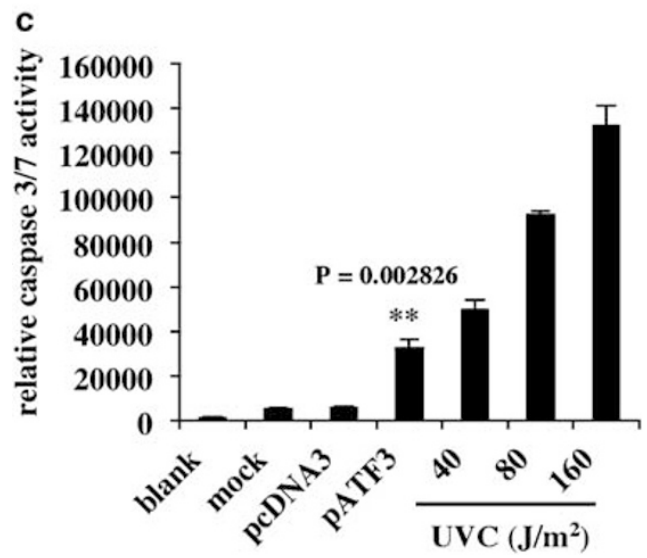

Figure 3 Overexpression of ATF3 triggers cell death. HeLa cells were transfected (transfection efficiency assessed using a GFP vector is about $60 \%$ ) with $0.5 \mu \mathrm{g}$ of a construct encoding ATF3. (a) Expression of exogenous ATF3 has been assessed by western blot. ERK corresponds to the loading control. (b) $24 \mathrm{~h}$ later, the number of living cells was assessed by trypan blue staining and (c) apoptosis was monitored by caspase 3/7 assay. The figures represent three separate experiments. Statistical analysis was performed (Student's $t$-test): ${ }^{\star \star} P$-value that represents a significant difference between pcDNA3- and pATF3-transfected cells

expression in UV-irradiated HaCaT, HeLa or ATF3-/-MEF cells led to a strong reduction in Hif-2alpha mRNA expression, thereby confirming our microarray data (Figure $4 \mathrm{c}$ and $\mathrm{d}$ ). In addition, transfection of increasing amount of ATF3-expressing construct in HeLa cells triggered a strong Hif-2alpha expression, as determined by QPCR (Figure 4e). These results showed that ATF3 expression was required and sufficient to promote Hif-2alpha transcripts. Interestingly, a canonical ATF-binding site (TGAGGTCA) is located within the $5^{\prime}$ part of the Hif-2alpha promoter at position -1615 from the transcription start site. To determine whether ATF3 acts through direct binding to Hif-2alpha regulatory sequences, we performed chromatin immunoprecipitation (ChIP) assay on UVC $\left(40 \mathrm{~J} / \mathrm{m}^{2}\right)$-irradiated HeLa cells followed by PCR using primers located in the promoter of Hif-2alpha. Significant PCR amplification, similar to the control genomic input and no ChIP condition, was observed for UVC-treated chromatin immunoprecipitated with anti-ATF3 antibodies 


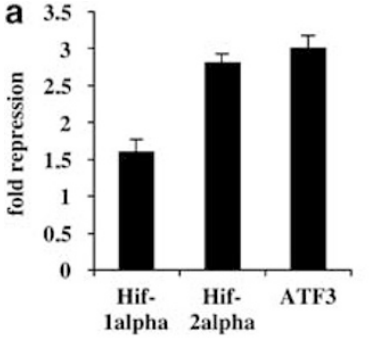

b
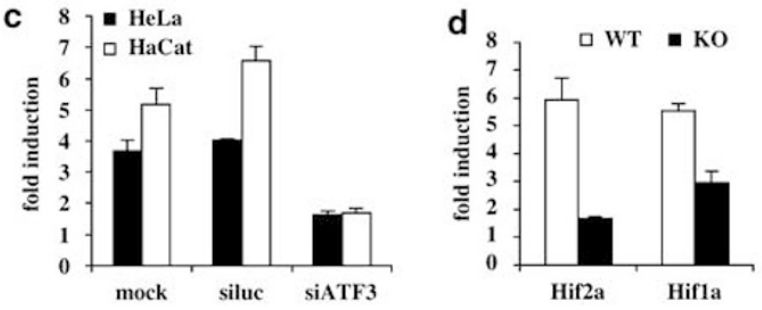

e

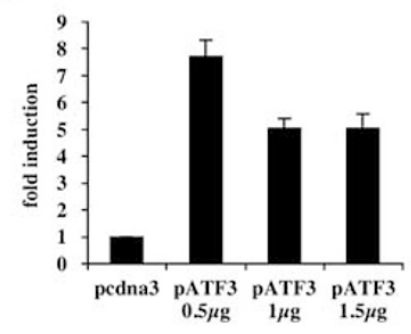

f

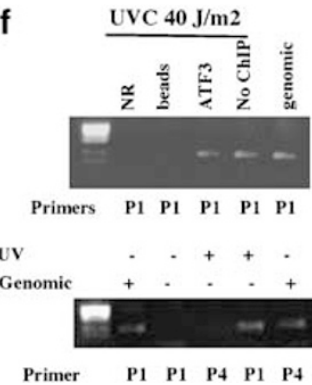

Figure 4 Hif-2alpha is a direct target gene of ATF3. (a) Human pan genomic microarray analysis was used to compare the transcriptome of UVC $\left(40 \mathrm{~J} / \mathrm{m}^{2}\right)$ irradiated HeLa cells rendered deficient in ATF3 expression with the transcriptome of UV-irradiated, ATF3-expressing cells. The figure shows Hif-1 and Hif-2alpha differential expression. (b) Time-course expressions of ATF3 and Hif-2alpha mRNA have been assessed by real-time PCR following UVC irradiation $\left(40 \mathrm{~J} / \mathrm{m}^{2}\right)$. Ct corresponds to the non-irradiated condition. (c) HeLa and $\mathrm{HaCaT}$ cells were transfected with siATF3(1) or siluc or not (mock). After $24 \mathrm{~h}$, cells were UVCirradiated $\left(40 \mathrm{~J} / \mathrm{m}^{2}\right)$ and total RNA was extracted $2 \mathrm{~h}$ after irradiation. Hif-2alpha mRNA expression was monitored by real-time RT-PCR. (d) Real-time RT-PCR analysis of Hif-1 and Hif-2alpha mRNA expression from total RNA extracted from UVC $\left(40 \mathrm{~J} / \mathrm{m}^{2}\right)$-irradiated ATF3 KO and WT MEF cells. (e) ATF3 overexpression is sufficient to stimulate Hif-2alpha. HeLa cells were transfected by increasing the amount of construction encoding ATF3. After $24 \mathrm{~h}$, total mRNA was extracted and Hif-2alpha expression was measured by real-time RT-PCR. The figures represent three separate experiments. (f) ATF3 physically interacts on Hif-2alpha promoter. ChIP experiments were performed using anti-ATF3 (ATF3) or non-relevant control anti-snap (NR) antibodies or agarose beads alone (beads) as described in Materials and Methods. No ChIP corresponds to a sample of prepared chromatin before immunoprecipitation. The last lane corresponds to control genomic DNA input. The detection of Hif-2alpha captured promoter was performed by PCR using the P1 primers located at the vicinity of an ATF-binding site located at position -1615 from the trascription start site (upper panel). PCR was performed on samples extracted from HeLa cells, UVC $\left(40 \mathrm{~J} / \mathrm{m}^{2}\right)$-irradiated or not, using the primers P1 or, as negative control, the non-relevant primers $\mathrm{P} 4$ located in a part of Hif-2alpha promoter that do not contain ATF-binding sites (lower panel). The first and last lanes correspond to control genomic DNA input

(Figure 4f). No amplification was observed with a nonrelevant serum or the control beads alone, or by using primers located in a part of the Hif-2alpha promoter that do not contain ATF-binding sites. These results demonstrate that ATF3 protein binds to the Hif-2alpha promoter within the chromatin context of UVC-treated epithelial cells. To evaluate whether the lack of Hif-2alpha mRNA has a repercussion on the protein expression level, protein extracts from UV-irradiated and control HaCaT cells used in Figure 2a were analyzed by western blotting (Figure 5a). In addition, the level of Hif-2alpha protein expression induced by UVC treatment was compared with the expression level obtained in hypoxic condition. The results showed that UVC irradiation induced the expression of Hif-2alpha protein only in cells expressing ATF3. Protein level in cells lacking ATF3 expression was similar to the control non-irradiated condition. Interestingly, blockade of transcription or protein translation with actinomycin $\mathrm{D}$ or cycloheximide, respectively, inhibited Hif-2alpha protein induction $5 \mathrm{~h}$ following UVC irradiation. This showed that Hif-2alpha expression upon UVC exposure required, at least at this time point, transcriptional and translational events (Figure 5b). Moreover, inhibition of JNK and P38 kinases that inhibited ATF3 expression (Figure 6a) also abolished Hif-2alpha expression (Figure 6b). Therefore, these two transcription factors share the same regulatory pathway. Taken together, our results demonstrate the major role of ATF3, under the control of JNK and P38 kinases, in the regulation of Hif-2alpha upon UV-mediated genotoxic stress.

Hif-2alpha is the death effector of ATF3. Since we found that the overexpression of ATF3 is sufficient to trigger apoptosis in HeLa cells (Figure $3 a$ and b), we sought to determine whether Hif-2alpha could also have a proapoptotic function. Therefore, we transiently transfected ATF3, Hif-2alpha-expressing constructs or an empty vector in HeLa cells. After $24 \mathrm{~h}$, proteins were extracted and subjected to immunoblotting using specific antibodies directed against the zymogen and cleaved forms of caspase-3. Overexpression of ATF3 and Hif-2alpha was controlled by western blot and real-time PCR (Supplementary data Figure $2 \mathrm{~A}$ and $\mathrm{B}$ ). We found that overexpression of Hif-2alpha as well as ATF3 was able to trigger the apoptotic process as seen by the detection of cleaved caspase-3, whereas almost undetectable level of cleaved caspase-3 was observed in the control empty vector (Figure 7a). The same experimental setting was used in the presence of an siRNA specific to Hif-2alpha (siHif2a(1)) or siLuc as control. siHif2a efficiency was monitored by realtime PCR (Supplementary data Figure 2A). Expression of both Hif-2alpha and ATF3 induced strong caspase 3/7 activity (mock and siLuc conditions; Figure 7b), which was severely reduced when Hif-2alpha expression was blocked (Figure 7b). To evaluate the contribution of Hif-2alpha in UV-induced apoptosis, we inhibited its expression in irradiated HeLa cells $\left(40 \mathrm{~J} / \mathrm{m}^{2}\right.$ UVC) and assessed both caspase activity (Figure 7c) and cell viability (Figure 7d). Although UVC induced strong caspase $3 / 7$ activity in the mock and siLuc control conditions, inhibition of Hif-2alpha as well as ATF3 expression by siHif2a(1) and siATF3(1), respectively, drastically reduced the apoptotic event and the number of dead cells. Same results were obtained using other siRNA sequences (siHif2a(2) and siATF3(2); Figure 7d). Therefore, lack of Hif-2alpha expression greatly promotes cell survival. To further confirm our results, we performed add back experiments by restoring ATF3 and 

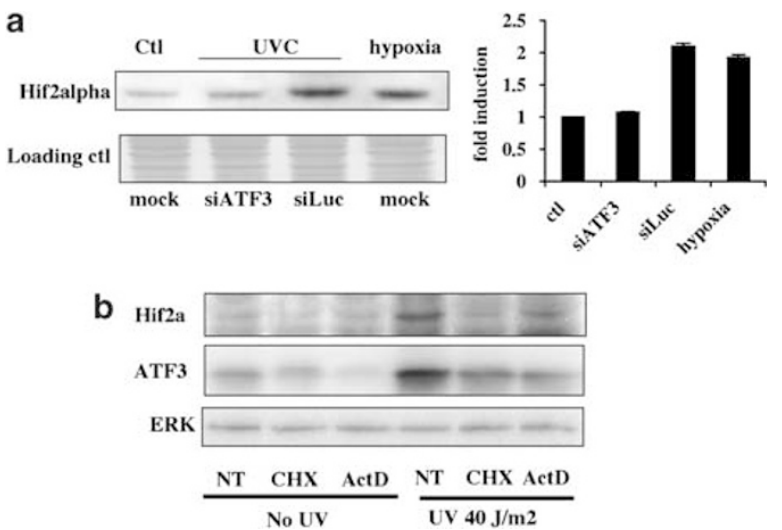

Figure 5 ATF3 is required for Hif-2alpha protein expression. (a) Western blot was performed using specific Hif-2alpha antibody in UVC-irradiated HaCaT cells transfected with the vehicle only (mock), siLuc or siATF3. The first and last lanes correspond to the normoxic non-irradiated condition (Ctl) and hypoxic control (hypoxia), respectively (left panel). Quantitation of protein expression level (Image $\mathrm{J}$ software). Fold induction corresponds to the ratio between non-irradiated normoxia and irradiated or hypoxia values. Normalizations were performed using the values of the loading control. Error bars represent the S.D. of three independent measures (right panel). (b) Hif-2alpha protein induction requires transcription and protein translation. $\mathrm{HaCaT}$ cells were incubated with cycloheximide and actinomycin D as described in Materials and Methods. Expression of Hif-2alpha and ATF3 protein was detected by western blot $5 \mathrm{~h}$ following UVC irradiation
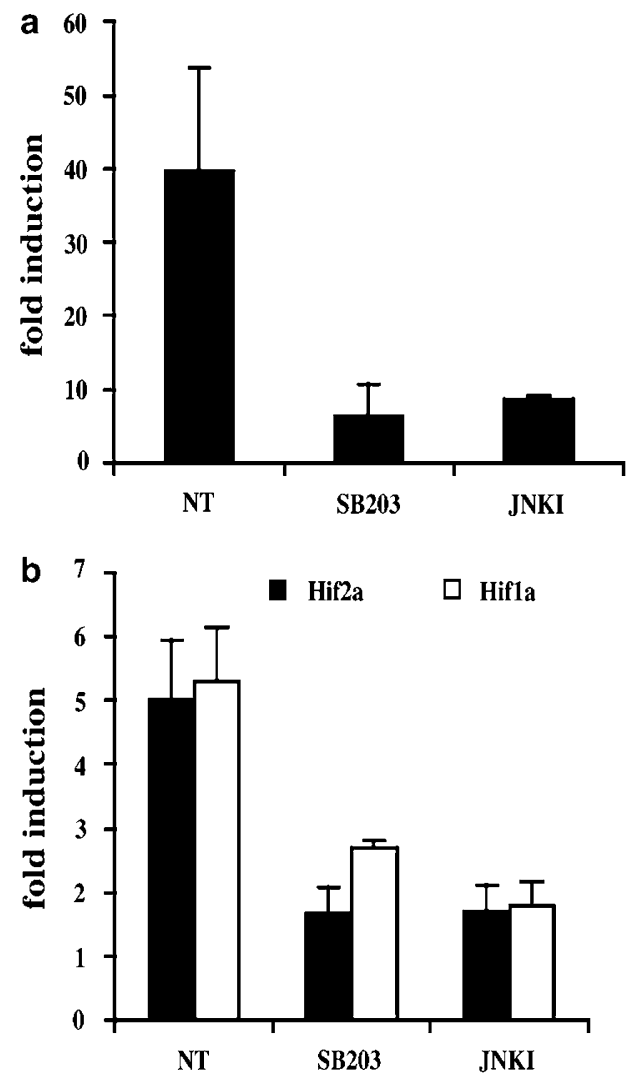

Figure 6 Expression of Hif-2alpha and ATF3 is dependent on stress-activated protein kinases pathway activation under UV irradiation. $\mathrm{HaCaT}$ cells were treated with $\mathrm{p} 38^{\mathrm{MAPK}}$ and JNK inhibitor or not (SB203, JNK1 and NT, respectively) $1 \mathrm{~h}$ before UVC irradiation $\left(40 \mathrm{~J} / \mathrm{m}^{2}\right) .2 \mathrm{~h}$ after irradiation, total RNA was extracted and expression of ATF3 (a) and Hif-2 or Hif-1alpha (b) was analyzed by real-time RT-PCR
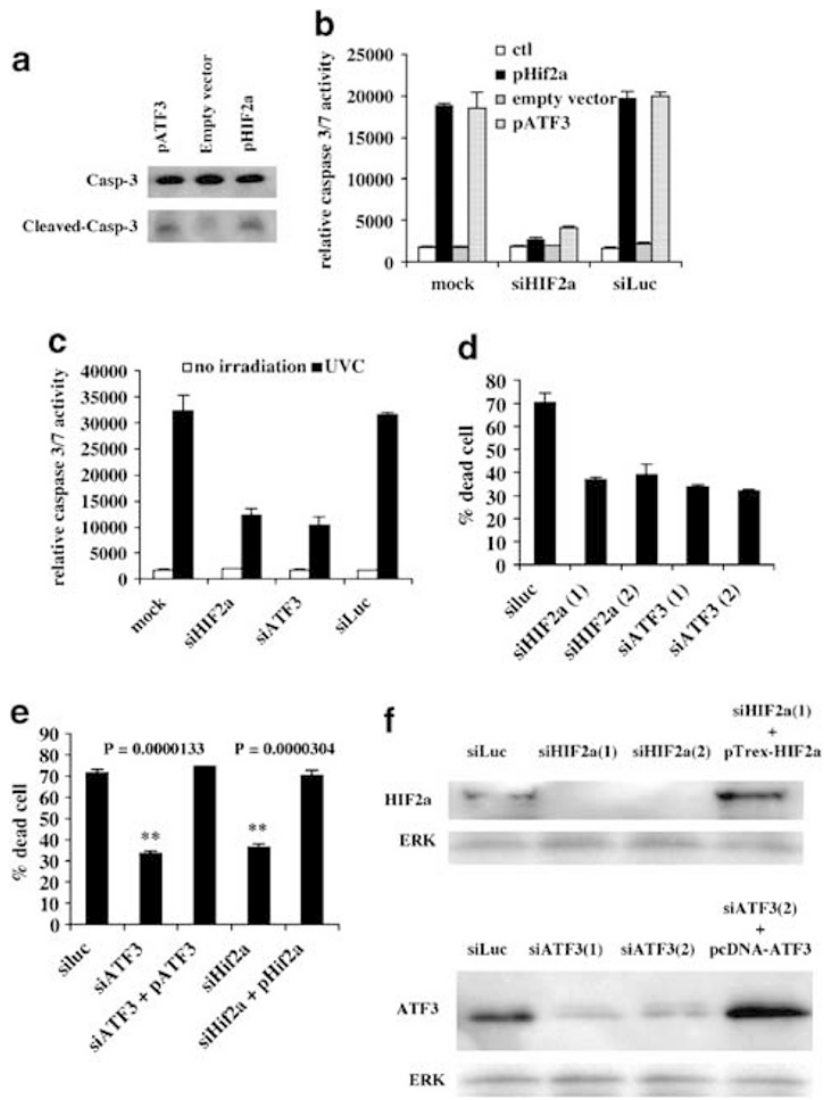

Figure 7 Hif-2alpha is the downstream effector of ATF3 in the mediation of UVinduced cell death. (a) Hif-2alpha overexpression induced apoptosis. HeLa cells were transfected by ATF3- or Hif-2alpha-encoding constructs. After $24 \mathrm{~h}$, protein was extracted and western blot analyses were performed with the caspase-3 and cleaved caspase-3 antibodies. (b) Hif-2alpha-mediated apoptosis was strongly repressed by siHif-2alpha. HeLa were co-transfected with siHif-2alpha(1) or siLuc or not (mock) and contructs encoding ATF3, Hif-2alpha or empty vector. After $24 \mathrm{~h}$, apoptosis was analyzed by caspase $3 / 7$ assay. (c) Hif-2alpha promotes UVCinduced apoptosis. HeLa were transfected with siHif-2alpha(1), siATF3(1) or siLuc or not (mock), $24 \mathrm{~h}$ before UVC irradiation $\left(40 \mathrm{~J} / \mathrm{m}^{2}\right)$. After $24 \mathrm{~h}$, apoptosis was monitored with caspase 3/7 assay. (d) HeLa cells were transfected with siATF3(1), siATF3(2), siHif2a(1), siHif2a(2) or siLuc as a control. After $24 \mathrm{~h}$, the cells were UVirradiated $\left(40 \mathrm{~J} / \mathrm{m}^{2}\right)$ and the percentage of dead cells was measured by trypan blue staining as described in Materials and Methods. (e) The same experimental setting was used to show the percentage of dead cells detected by trypan blue staining. Statistical analysis was performed (Student's $t$-test): ${ }^{* \star} P$-value that represents a significant difference between siLuc- and siATF3- or siLuc- and siHif2a-treated cells. Add back experiments were performed by the transfection of contructs encoding ATF3 and Hif-2alpha in cells rendered deficient in both proteins by the use of siATF3(2) and siHif-2alpha(1). (f) Efficiency of siHif2a(1), siHif2a(2) and Hif-2alpha protein rescue as well as siATF3(1), siATF3(2) and ATF3 protein rescue were monitored by western blot as described in Materials and Methods

Hif-2alpha expression using insensitive cDNA in siHif2a(1)and siATF3(2)-transfected HeLa cells. As expected, gain of ATF3 as well as Hif-2alpha expression totally rescued UVinduced cell death (Figure 7e). Efficiency of the expression of both proteins has been assessed by western blot (Figure 7f). Taken together, our results demonstrate Hif-2alpha as a major effector of ATF3 in mediating UV-dependent cell death. 
Hif-2alpha is a major mediator of UV-induced apoptosis. Hif-1alpha is known as a proapoptotic effector of UV-induced cell death in keratinocytes. ${ }^{21}$ We have demonstrated in Figure $7 \mathrm{c}$ and $\mathrm{d}$ that inhibition of Hif2alpha expression drastically impaired UV-induced apoptosis, suggesting that Hif-2alpha is a better death effector than Hif-1alpha upon UV-mediated genotoxic stress. To test this hypothesis, we compared the amount of cleaved caspase-3 in UV-irradiated HaCaT cells transfected with specific siRNA to Hif-1alpha (siHIF1a), siHif2a(1) or siluc as control. The specificity and efficiency of each siRNA were controlled by QPCR analysis of Hif-1 and Hif-2alpha mRNA expression. As seen in Figure $8 a$, both siRNAs induced strong and specific RNA interference. Treated $\mathrm{HaCaT}$ cells were therefore subjected to a caspase $3 / 7$ assay. As shown in Figure $8 b$, strong caspase $3 / 7$ activity was observed only in UV-irradiated cells (siLuc control lanes). Under these UV-induced apoptotic conditions, inhibition of Hif2alpha expression led to the strongest reduction (about 63\%) of caspase 3/7 activation as compared with lack of Hif-1alpha expression (about 44\%) (Figure 8b). Although Hif-1alpha clearly contributes to apoptosis, Hif-2alpha appears to be more efficient and is therefore one of the major mediators of apoptosis under UVirradiated conditions in this cell line.

Both Hif-2 and Hif-1alpha stimulate the expression of proapoptotic genes. To delineate the respective ability

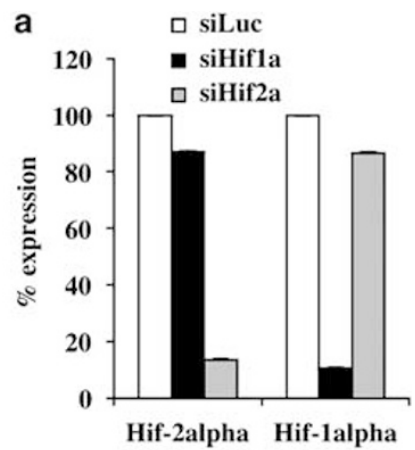

b



Figure 8 Hif-2alpha is a major mediator of UV-induced apoptosis. HaCaT cells were transfected either with siHif1a, siHif2a(1) or siLuc as a control. After $24 \mathrm{~h}$, the cells were UV-irradiated $\left(40 \mathrm{~J} / \mathrm{m}^{2}\right)$ or not (Ctl). After $24 \mathrm{~h}$, expression of Hif-2 and Hif1alpha mRNA was assessed by real-time RT-PCR (a), assessment of caspase activity was performed as described in Materials and Methods (b). Statistical analysis was performed (Student's $t$-test); ${ }^{\star \star} P$-value that represents a significant difference between siLuc- and siHif1a- or siLuc- and siHif2a-treated cells of Hif-2 and Hif-1alpha to mediate cell death, we have compared the mRNA expression of a panel of 88 pro- or antiapoptotic genes in $\mathrm{HaCaT}$ cells rendered deficient either in Hif-1 or Hif-2alpha expression (Figure 8a). Gene expression was determined by QPCR using specific primers distributed in 96-well plates. HaCaT cells were transfected with siHif1a, siHif2a(1) or siLuc. Inhibition of Hif-1 as well as Hif-2alpha led to a significant decrease in the expression of proapoptotic genes, such as Caspase7 and TRAIL, which displayed at least two and fourfold repression, respectively (Table 1 ). Although some of them were found to be upregulated by both Hif-1 and Hif-2alpha (i.e., downregulated by knockdown of Hif genes), others were specific to each gene. This was the case of BNIP3 (BCL2/ adenovirus $\mathrm{E} 1 \mathrm{~B} 19 \mathrm{kDa}$ interacting protein 3 ), which was exclusively regulated by Hif-1alpha or EGR2 and deathassociated protein kinase 3 whose regulation was dependent only on Hif-2alpha. Most of these genes, except EGR1 and BNIP3, are also downregulated by the knockdown of ATF3 (Table 1). These results indicate that Hif genes are able to promote apoptosis by contributing to the expression of proapoptotic genes.

\section{Discussion}

In the present study, we describe a novel ATF3-dependent cell death pathway, triggered by the activation of $\mathrm{p} 38$ and JNK, in UV-stressed epithelial cells. We provide evidences that ATF3 contributes to UV-induced apoptosis through the regulation of Hif-2alpha, which in turn contributes to the expression of proapoptotic genes, such as TRAIL (Figure 9).

Our results demonstrate the requirement of ATF3 expression to initiate appropriate programmed cell death following UV stress. Indeed, UV-irradiated ATF3-null mouse embryonic fibroblasts or cells in which ATF3 expression has been suppressed are systematically less sensitive to death. Interestingly, knockdown of ATF3 protein leads to $50 \%$ decrease in caspase activity. This indicates that ATF3 belongs to a group of important mediators of UV-induced apoptosis that are required together to ensure maximal caspase activation. Conversely, gain of function of ATF3 is

Table $1 \mathrm{Hif}-1$ and Hif-2alpha contribute to the expression of proapoptotic genes

\begin{tabular}{lccc}
\hline Knockdown & Hifa2a & Hifa1a & ATF3 \\
\hline EGR1 & -1.6 & -2 & +2 \\
DAPK3 & -1.8 & 1 & -2.6 \\
GADD45 & -1.9 & -1.5 & -2.4 \\
FKHR2 & -1.8 & -1.8 & -1.5 \\
EGR2 & -1.9 & 1 & -2.9 \\
Casp7 & -2.5 & -2 & -1.8 \\
TRAIL & -4.5 & -4 & -3.6 \\
BNIP3 & 1 & -2 & 1.4 \\
\hline
\end{tabular}

ATF3, activating transcription factor 3; BNIP3, BCL2/adenovirus E1B $19 \mathrm{kDa}$ interacting protein 3; Casp7, Caspase7; DAPK3, death-associated protein kinase 3; EGR, early growth response; GADD45, growth arrest and DNAdamage-inducible; Hif, hypoxia inducible factor; TRAIL, tumor necrosis factor (ligand) superfamily (member 10). The table represents the fold repression (QPCR) of proapoptotic genes that are downregulated when the expression of Hif-1 or Hif-2alpha is inhibited. The third column corresponds to the fold repression or induction assessed in our microarray analysis of ATF3 knockdown upon UV exposure 


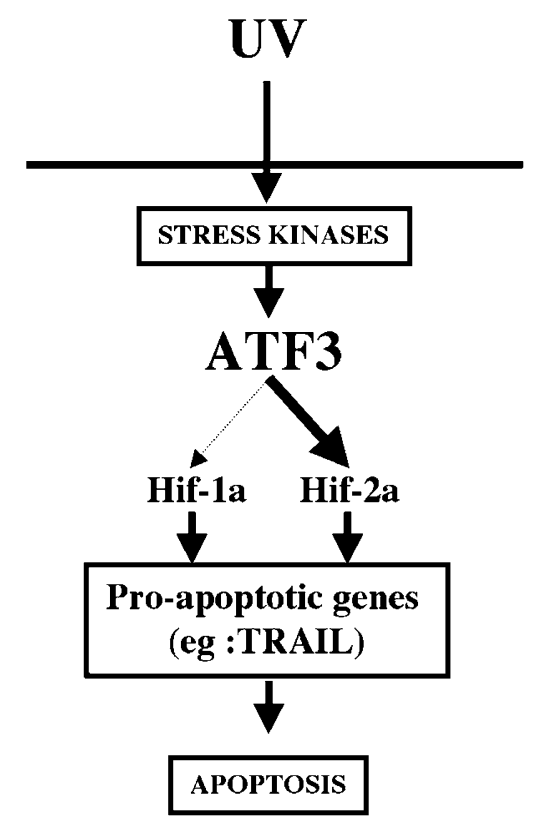

Figure 9 A novel cell death pathway dependent on ATF3 expression. Upon stress, P38 and JNK activate ATF3 gene expression, which in turn induces the expression of both Hif-2 and Hif-1alpha. Those genes are necessary for UVmediated apoptosis by contributing to the expression of proapoptotic genes such as TRAIL. The dashed arrow represents partial regulation of Hif-1alpha expression by ATF3

sufficient to trigger apoptosis in epithelial cultured cells. Other experimental studies have shown ATF3 contribution to UV, curcumin or LY294002-mediated apoptosis. ${ }^{17,18,22}$ ATF3 has been further demonstrated to be a novel regulator of stressinduced $\beta$-cell apoptosis in a JNK and NF-kB pathwaydependent manner. ${ }^{14}$ Accordingly, our results strongly sustain the proapoptotic role of ATF3 in UV-induced stress response. However, reports describe a protective role of ATF3 in biological process such as acute neuronal injury models, ${ }^{23}$ and an opposite effect has also been reported in two models of TNFalpha-induced endothelial cell death. ${ }^{24,25}$ Although such functional discrepancies remain to be investigated, it is reasonable to speculate that the physiological context, the time course and the level of ATF3 expression would determine the set of target effectors responsible for its functions. In this particular context, we have demonstrated that ATF3 is responsible for Hif-2alpha expression. The proapoptotic function of Hif-2alpha has been described in several biological contexts, such as hypoglycemia of ES cells, human malignant glioblastoma or in placenta from pregnancies with fetal growth restriction. ${ }^{26-28}$ This proapoptotic function confers on Hif-2alpha a tumor suppressor's property, despite its well-known angiogenic role. Our results sustain its tumor suppressor function as suppression of Hif-2alpha protects the cells from UV-induced apoptosis, whereas gain of function is sufficient to trigger epithelial cell death with strong caspase 3/7 activation. The proapoptotic effects of ATF3 and Hif-2alpha are clearly correlated, and inhibition of HIF-2alpha expression strongly impairs ATF3-mediated cell death. Thus, Hif-2alpha constitutes the major effector of ATF3 in mediating UV-induced apoptosis. The major role of Hif2alpha in such a process is further underlined by the fact that Hif-1alpha, already known as a key apoptotic factor in the keratinocyte response to $\mathrm{UV},{ }^{21}$ is not able to rescue loss of apoptosis due to the lack of Hif-2alpha expression. We have further demonstrated that Hif-2 and Hif-1alpha are involved in the upregulation of proapoptotic genes such as Caspase7 or TRAIL. Interestingly, it has been reported that one of the effects of UV irradiation is to reduce the number of TRAIL decoy receptors. This is in favor of an increase in the number of TRAIL and its death receptor interactions, thereby promoting the susceptibility of epithelial cells to TRAILinduced apoptosis. ${ }^{29}$ Impaired apoptosis due to lack of Hif genes may indeed be explained by the inhibition of TRAIL expression. We have therefore identified an important cascade of gene activation that provides new insights into the mechanism by which ATF3 may act as a proapoptotic factor. It has been described that ATF3 mediates cell death through a non-transcriptional mechanism, as it contributes, upon genotoxic stress, to $\mathrm{p} 53$ protein stabilization by physical association, thereby preventing its MDM2-mediated degradation. ${ }^{30}$ However, we demonstrate here that the apoptotic process following DNA damage is impaired in HaCaT cells rendered deficient in ATF3 expression, and in which the active form of $\mathrm{p53}$ is mutated. Furthermore, Hif-2alpha as an apoptotic effector demonstrates that ATF3 promotes its proapoptotic function through transcriptional activity. ATF3 therefore plays an important role in UV-induced apoptosis, as it is able to mediate death through different mechanisms.

The cellular genotoxic stress response is considered as a protective mechanism leading to the successful repair or death of severely damaged cells, thereby constituting an inducible anticancer barrier., ${ }^{1,2}$ Alterations that compromise the DNA damage response might allow cell proliferation, survival, genomic instability and thus tumor progression. Efficient regulation of DNA repair and cell death in such a process is crucial. Our results show that ATF3, through the induction of Hif-2alpha, plays an important role in UV-induced apoptosis of damaged epithelial cells. Therefore, ATF3 as well as Hif-2alpha may be considered as important early regulators of epithelial homeostasis during genotoxic stress response. Although several reports suggest a procancerous function for ATF3, ${ }^{16,31}$ a growing body of recent evidences argues for an antagonistic role in human cancer. Gene expression patterns in various human cancers, including lung, breast, kidney, liver, colon and prostate, reveal downregulation of ATF3 expression. ${ }^{32-38}$ In addition, recent cancer profiling array studies that compare 154 microdissected human tumors and their corresponding normal tissues, as well as the conclusions of the Cancer Genome Anatomy Project (http://www.cgap.nci.nih.gov), ${ }^{39}$ confirm that the overall expression level of ATF3 in cancers is statistically lower than in normal tissues. It is therefore tempting to speculate that impaired apoptosis due to lack of ATF3 and Hif-2alpha induction, during the DNA damage response, may be, at least in part, responsible for oncogenesis by allowing the accumulation of cells bearing severe chromosomic alterations. Accordingly, ATF3 and Hif-2alpha may be considered as potent tumor suppressors in epithelial cells and efficient gatekeepers of the anticancer barrier. 


\section{Materials and Methods}

Cell culture. Cells were cultivated at $37^{\circ} \mathrm{C}$ in $5 \% \mathrm{CO}_{2}$. MEFs derived from ATF3-null mice were obtained from Tsonwin Hai (Department of Molecular and Cellular Biochemistry, Center for Molecular Neurobiology, Ohio State University, $\mathrm{OH}, \mathrm{USA}$ ). ${ }^{18}$ Immortalized keratinocytes (HaCaT cells), HeLa and MEFs were maintained in Dulbecco's modified Eagles' medium supplemented with $10 \%$ bovine fetal serum. When indicated, the cells received a single dose of $40 \mathrm{~J} / \mathrm{m}^{2}$ UVC. To inhibit the MAPK, PI3K, P38 and JNK pathways, the cells were incubated, $1 \mathrm{~h}$ prior to treatment, with $15 \mu$ M each of U0126, LY294002, SB203580, JNK inhibitor II and $10 \mu \mathrm{M}$ Wortmannin (VWR). To inhibit transcription and protein translation, cells were incubated with $10 \mu \mathrm{g} / \mathrm{ml}$ actinomycin D or cycloheximide $1 \mathrm{~h}$ prior to UVC irradiation $\left(40 \mathrm{~J} / \mathrm{m}^{2}\right)$

Apoptosis and cell death measurement. Cell death measurement was performed $24 \mathrm{~h}$ following UVC irradiation by trypan blue staining. The cells were counted using a malassez counting chamber. Experiments were repeated at least three times and the cells were counted four independent times. The detection of apoptosis was performed by measurement of caspase-3 and 7 activity using the Caspase-Glo ${ }^{\mathrm{TM}} 3 / 7$ Assay according to the manufacturer's protocol (Promega).

Plasmids. Activating transcription factor 3 promoter cloned into PGL3basic and ATF3-expressing vector was provided by Dr. Shigetaka Kitajima. ${ }^{40}$ Hif-2alphaexpressing construct was provided by Dr. Nathalie Mazure (Institute of Signaling, Developmental Biology and Cancer Research, CNRS UMR 6543, University of Nice, France).

\section{Transient transfection}

Luciferase assay. The day before transfection, HeLa cells were seeded in 24well plates at a density of 60000 cells per well. The cells were transfected with Lipofectamine 2000 reagent (Invitrogen) according to the manufacturer's instructions. The day after transfection, cells were UVC-irradiated $\left(40 \mathrm{~J} / \mathrm{m}^{2}\right)$ and, after $4 \mathrm{~h}$, luciferase activity was measured using a microlumat LB96P (EG\&G Berthold). The results are the average of three separate experiments in quadruplicate.

siRNA transfection. HaCaT or HeLa cells were plated 1 day before transfection in $60 \mathrm{~mm}$ wells at a density of 800000 cells per dish. Transfection of siRNA was performed using Lipofectamine 2000 reagent $(0.1 \mu \mathrm{M}$ siRNA with $20 \mu \mathrm{l}$ lipofectamine). The sequences of siATF3(1) and SiATF3(2) are, respectively, GCACCUCUGCCACCGGAUGdTdT and GCAGAAAGUUCAACUUCCAdTdT The sequences of siHif2a(1), siHif2a(2) and siHif1a are, respectively, CAGCAUCUUUGAUAGCAGUdTdT, CCCUAUAUCCCCAUGGACGGdTdT and CUGAUGACCAGCAACUUGAdTdT. These siRNAs were provided by Dr. Nathalie Mazure.

Plasmid transfection was performed using Lipofectamine 2000 reagent according to the manufacturer's instructions (Invitrogen). Add back experiments were performed using siATF3(2) directed against the $3^{\prime}$-UTR part of ATF3 mRNA and a cDNA construct expressing only the coding sequence of ATF3. To create mismatches with siHif2a(1), the Hif-2alpha-expressing construct was mutated without changing the amino-acid sequence using the Quickchange technique according to the manufacturer's instruction (Stratagene). Mutagenesis was performed using the following mutated primers: Hif2alphamut-L 5'-CACCTGATGGCCATGAACTCTATTTTCGACTCTTCCGGCAAGGGGGCTGT

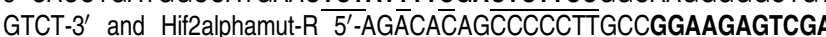
AAATAGAGTTCATGGCCATCAGGTG-3'. The bases in bold correspond to the sequence targeted by the siRNA and the bases underlined indicate the mutations.

Chromatin immunoprecipitation assay. Live cells were treated with $1 \%$ formaldehyde for $5 \mathrm{~min}$ at $37^{\circ} \mathrm{C}$ and $30 \mathrm{~min}$ at $4^{\circ} \mathrm{C}$. The cells were then harvested and suspended in lysis buffer ( $10 \mathrm{mM} \mathrm{HEPES}, 1.5 \mathrm{mM} \mathrm{MgCl}_{2}, 10 \mathrm{mM} \mathrm{KCl}, 0.5 \mathrm{mM}$ DTT, $0.1 \%$ NP40 and protease inhibitors). After 10 min incubation on ice, the cells were centrifuged ( 5000 r.p.m., $5 \mathrm{~min}$ at $4^{\circ} \mathrm{C}$ ) to pellet the nuclei. The nuclei were then suspended in nuclei lysis buffer $(50 \mathrm{mM}$ Tris-Cl $(\mathrm{pH} 8.1), 10 \mathrm{mM}$ EDTA, $1 \%$ SDS and protease inhibitors) and incubated on ice for $10 \mathrm{~min}$. The chromatin was sheared by sonication (average size of $1 \mathrm{~kb}$ ) and samples were microfuged at 14000 r.p.m. for $10 \mathrm{~min}$. The sheared chromatin was then immunoprecipitated using specific antibody directed against ATF3 (Santa Cruz Biotechnology Inc. Santa Cruz (sc-188), CA, USA), and a non-relevant antibody directed against snap protein as the negative control. After immunoprecipitation, the crosslink was reverted by heat treatment $\left(67^{\circ} \mathrm{C}\right.$ overnight and proteinase $\mathrm{K}$ digestion). The genomic captured fragments were then recovered by phenol-chloroform extraction. The captured regulatory sequences were identified by PCR analysis using appropriate primers located at the vicinity of a canonical ATF-binding site (TGAGGTCA, position -1615 from the transcription start) within Hif-2alpha regulatory sequences (1.7 kb upstream of transcription start site):

PHEPAS1-p1L-CHIP: 5'-TTGAGAGGCCAAGTTGGGCAGA-3' (position -1641 from the transcription start site);

PHEPAS1-p1R-CHIP: 5'-GCCCACCACCACCCTCAGCTA-3' (position -1464 from the transcription start site).

The negative control was performed using primers located in an irrelevant part of Hif-2alpha promoter that do not contain ATF-binding sites:

PHEPAS1-p4L-CHIP: 5'-ACGACCTCATAAACAAGTCC-3' (position -289 from the transcription start site);

PHEPAS1-p4L-CHIP: 5'-ACCGGCGCGGGTGTGTGG-3' (position -100 from the transcription start site).

Quantitative real-time one-step RT-PCR and western blot. RNAs were extracted using Trizol reagent (Invitrogen) and Rneasy protect mini kit (Qiagen). mRNA expression levels were quantified by real-time one-step RT-PCR using the SYBR-Green PCR Master Mix (Eurogentec or Applied Biosystems) according to the manufacturer's instructions. The expression of each gene was determined relative to GAPDH as an internal control, and the fold stimulation was calculated by using the equation $2^{-\Delta \Delta C T}$, where $\Delta C T=\mathrm{CT}_{\text {gene }}-\mathrm{CT}_{\text {GAPDH }}$ and $\Delta \Delta C T=\Delta C T_{\text {stimulated condition }}-\Delta \mathrm{CT}_{\text {unstimulated condition. }}$ Each gene was amplified using the following appropriate specific primers: human-ATF31002F-AGCAGGCCCTTCCCATTC; human-ATF3-1068R-AGTTGAGGCAAAGA TG;CACTTG; human-HIF2aF-GCGCTAGACTCCGAGAACAT; human-HIF2a-RTGGCCACTTACTACCTGACCCTT. Specific primers to Hif-1alpha were provided by Dr. Nathalie Mazure and the sequences are available upon request (Nathalie.MAZURE@unice.fr).

Detection of genes linked to apoptosis was performed in a 96-well plate format designed and provided by Dr. Marcel Decker (INSERM U576, Nice F-06202; Marcel.DECKERT@unice.fr).

For the western blot analysis, total protein was extracted 2 or $3 \mathrm{~h}$ after irradiation with buffer containing the following: $20 \mathrm{mM}$ HEPES, $350 \mathrm{mM} \mathrm{NaCl}, 500 \mu \mathrm{M}$ EDTA, $1 \mathrm{mM} \mathrm{MgCl} 220 \%$ glycerol and $1 \% \mathrm{NP} 40$. The extract was then boiled and separated on SDS-polyacrylamide gel electrophoresis. The proteins were transferred to Immobilon P membranes (Millipore Corporation, Bedford, MA, USA) and probed with primary rabbit polyclonal antibodies to ATF3 (Santa Cruz Biotechnology (sc188) Inc.) or to Hif-2alpha (provided by Dr. Nathalie Mazure) mouse monoclonal antibody to $\alpha$ ERK2 D-2 (Santa Cruz Biotechnology Inc. (sc-1647)). Blots were subsequently probed with anti-rabbit or anti-mouse secondary antibodies conjugated to horseradish peroxidase for enhanced chemiluminescence detection of the signals (Amersham, Grand Island, NY, USA).

Acknowledgements. We thank Tsonwin Hai (Department of Molecular and Cellular Biochemistry Center for Molecular Neurobiology) for the gift of the immortalized ATF3-/-mouse embryonic fibroblast and matching wild-type cells. We would also like to thank Dr. Pascal Barbry and Virginie Virolle (IPMC, Sophia Antipolis, France) for the supply and hybridization of human pangenomic microarrays. This work was supported by grants from Association pour la Recherche sur le Cancer (ARC), Société de Recherche en Dermatologie (SRD), Institut National de la Santé et de la Recherche Medicale (INSERM).

1. Bartkova J, Horejsi Z, Koed K, Kramer A, Tort F, Zieger $K$ et al. DNA damage response as a candidate anti-cancer barrier in early human tumorigenesis. Nature 2005; 434: 864-870.

2. Gorgoulis VG, Vassiliou LV, Karakaidos $P$, Zacharatos $P$, Kotsinas A, Liloglou T et al. Activation of the DNA damage checkpoint and genomic instability in human precancerous lesions. Nature 2005; 434: 907-913.

3. Brash D, Ponten J. Skin precancer. Cancer Surv 1998; 32: 69-113

4. Brash DE. Sunlight and the onset of skin cancer. Trends Genet 1997; 13: 410-414.

5. Thyss R, Virolle V, Imbert V, Peyron JF, Aberdam D, Virolle T. NF-kappaB/Egr-1/Gadd45 are sequentially activated upon UVB irradiation to mediate epidermal cell death. EMBO J 2005; 24: 128-137. epub 2004 Dec 16

6. Virolle T, Adamson ED, Baron V, Birle D, Mercola D, Mustelin T et al. The Egr-1 transcription factor directly activates PTEN during irradiation-induced signalling. Nat Cell Biol 2001; 3: 1124-1128. 
7. Dazard JE, Gal H, Amariglio N, Rechavi G, Domany E, Givol D. Genome-wide comparison of human keratinocyte and squamous cell carcinoma responses to UVB irradiation implications for skin and epithelial cancer. Oncogene 2003; 22: 2993-3006.

8. Kool J, Hamdi M, Cornelissen-Steijger $P$, van der Eb AJ, Terleth C, van Dam H Induction of ATF3 by ionizing radiation is mediated via a signaling pathway that includes ATM, Nibrin1, stress-induced MAPkinases and ATF-2. Oncogene 2003; 22 4235-4242.

9. Hai T, Hartman MG. The molecular biology and nomenclature of the activating transcription factor/cAMP responsive element binding family of transcription factors: activating transcription factor proteins and homeostasis. Gene 2001; 273: 1-11.

10. Liang G, Wolfgang CD, Chen BP, Chen TH, Hai T. ATF3 gene. Genomic organization, promoter, and regulation. J Biol Chem 1996; 271: 1695-1701.

11. Lin YS, Green MR. Interaction of a common cellular transcription factor, ATF, with regulatory elements in both E1a- and cyclic AMP-inducible promoters. Proc Natl Acad Sci USA 1988; 85: 3396-3400.

12. Fan $\mathrm{F}$, Jin $\mathrm{S}$, Amundson $\mathrm{SA}$, Tong $\mathrm{T}$, Fan $\mathrm{W}$, Zhao $\mathrm{H}$ et al. ATF3 induction following DNA damage is regulated by distinct signaling pathways and over-expression of ATF3 protein suppresses cell growth. Oncogene 2002; 21: 7488-7496.

13. Harper EG, Alvares SM, Carter WG. Wounding activates p38 map kinase and activation transcription factor 3 in leading keratinocytes. J Cell Sci 2005; 118: 3471-3485.

14. Hartman MG, Lu D, Kim ML, Kociba GJ, Shukri T, Buteau J et al. Role for activating transcription factor 3 in stress-induced beta-cell apoptosis. Mol Cell Biol 2004; 24 $5721-5732$.

15. Hai T, Wolfgang CD, Marsee DK, Allen AE, Sivaprasad U. ATF3 and stress responses. Gene Expr 1999; 7: 321-335.

16. Ishiguro $T$, Nagawa $H$, Naito $M$, Tsuruo $T$. Inhibitory effect of ATF3 antisense oligonucleotide on ectopic growth of HT29 human colon cancer cells. Jpn J Cancer Res 2000; 91: 833-836.

17. Yan C, Jamaluddin MS, Aggarwal B, Myers J, Boyd DD. Gene expression profiling identifies activating transcription factor 3 as a novel contributor to the proapoptotic effect of curcumin. Mol Cancer Ther 2005; 4: 233-241.

18. Lu D, Wolfgang CD, Hai T. Activating transcription factor 3 , a stress-inducible gene suppresses Ras-stimulated tumorigenesis. J Biol Chem 2006; 281: 10473-10481.

19. Averous J, Bruhat A, Jousse C, Carraro V, Thiel G, Fafournoux P. Induction of $\mathrm{CHOP}$ expression by amino acid limitation requires both ATF4 expression and ATF2 phosphorylation. J Biol Chem 2004; 279: 5288-5297.

20. Le Brigand K, Russell R, Moreilhon C, Rouillard JM, Jost B, Amiot F et al. An open-access long oligonucleotide microarray resource for analysis of the human and mouse transcriptomes. Nucleic Acids Res 2006; 34: e87.

21. Rezvani HR, Dedieu S, North S, Belloc F, Rossignol R, Letellier T et al. Hypoxia-inducible factor-1alpha, a key factor in the keratinocyte response to UVB exposure. $\mathrm{J}$ Biol Chem 2007; 282: 16413-16422.

22. Yamaguchi K, Lee SH, Kim JS, Wimalasena J, Kitajima S, Baek SJ. Activating transcription factor 3 and early growth response 1 are the novel targets of LY294002 in a phosphatidylinositol 3-kinase-independent pathway. Cancer Res 2006; 66 2376-2384.

23. Nakagomi S, Suzuki Y, Namikawa K, Kiryu-Seo S, Kiyama H. Expression of the activating transcription factor 3 prevents c-Jun $\mathrm{N}$-terminal kinase-induced neuronal death by promoting heat shock protein 27 expression and Akt activation. J Neurosci 2003; 23 : 5187-5196.

24. Kawauchi J, Zhang C, Nobori K, Hashimoto Y, Adachi MT, Noda A et al. Transcriptional repressor activating transcription factor 3 protects human umbilical vein endothelial cells from tumor necrosis factor-alpha-induced apoptosis through down-regulation of p53 transcription. J Biol Chem 2002; 277: 39025-39034.

25. Nawa T, Nawa MT, Adachi MT, Uchimura I, Shimokawa R, Fujisawa K et al. Expression of transcriptional repressor ATF3/LRF1 in human atherosclerosis: colocalization and possible involvement in cell death of vascular endothelial cells. Atherosclerosis 2002; 161: 281-291.

26. Acker T, Diez-Juan A, Aragones J, Tjwa M, Brusselmans K, Moons L et al. Genetic evidence for a tumor suppressor role of HIF-2alpha. Cancer Cell 2005; 8: 131-141.

27. Brusselmans K, Bono F, Maxwell P, Dor Y, Dewerchin M, Collen D et al. Hypoxia-inducible factor-2alpha (HIF-2alpha) is involved in the apoptotic response to hypoglycemia but not to hypoxia. J Biol Chem 2001; 19: 39192-39196.

28. Dai S, Kanenishi K, Ueno M, Sakamoto H, Hata T. Hypoxia-inducible factor-2alpha is involved in enhanced apoptosis in the placenta from pregnancies with fetal growth restriction. Pathol Int Nov 2004; 54: 843-849.

29. Qin JZ, Bacon P, Panella J, Sitailo LA, Denning MF, Nickoloff BJ. Low-dose UV-radiation sensitizes keratinocytes to TRAIL-induced apoptosis. J Cell Physiol 2004; 200: 155-166.

30. Yan C, Lu D, Hai T, Boyd DD. Activating transcription factor 3 , a stress sensor, activates p53 by blocking its ubiquitination. EMBO $\mathrm{J} 2005 ; 24$ : 2425-2435.

31. Ishiguro T, Nakajima M, Naito M, Muto T, Tsuruo T. Identification of genes differentially expressed in B16 murine melanoma sublines with different metastatic potentials. Cancer Res 1996; 56: 875-879.

32. Beer DG, Kardia SL, Huang CC, Giordano TJ, Levin AM, Misek DE et al. Gene-expression profiles predict survival of patients with lung adenocarcinoma. Nat Med 2002; 8: 816-824.

33. Chen X, Cheung ST, So S, Fan ST, Barry C, Higgins J et al. Gene expression patterns in human liver cancers. Mol Biol Cell 2002; 13: 1929-1939.

34. Higgins JP, Shinghal R, Gill H, Reese JH, Terris M, Cohen RJ et al. Gene expression patterns in renal cell carcinoma assessed by complementary DNA microarray. Am J Pathol 2003; 162: 925-932.

35. Lapointe J, Li C, Higgins JP, van de Rijn M, Bair E, Montgomery K et al. Gene expression profiling identifies clinically relevant subtypes of prostate cancer. Proc Natl Acad Sci USA 2004; 101: 811-816.

36. Notterman DA, Alon U, Sierk AJ, Levine AJ. Transcriptional gene expression profiles of colorectal adenoma, adenocarcinoma, and normal tissue examined by oligonucleotide arrays. Cancer Res 2001; 61: 3124-3130.

37. Perou CM, Jeffrey SS, van de Rijn M, Rees CA, Eisen MB, Ross DT et al. Distinctive gene expression patterns in human mammary epithelial cells and breast cancers. Proc Natl Acad Sci USA 1999; 96: 9212-9217.

38. Sorlie T, Perou CM, Tibshirani R, Aas T, Geisler S, Johnsen H et al. Gene expression patterns of breast carcinomas distinguish tumor subclasses with clinical implications. Proc Natl Acad Sci USA 2001; 98: 10869-10874.

39. Yan C, Boyd DD. ATF3 regulates the stability of p53: a link to cancer. Cell Cycle 2006; 5 926-929.

40. Cai Y, Zhang C, Nawa T, Aso T, Tanaka M, Oshiro S et al. Homocysteine-responsive ATF3 gene expression in human vascular endothelial cells: activation of c-Jun $\mathrm{NH}(2)$-terminal kinase and promoter response element. Blood 2000; 96: 2140-2148.

\section{Supplementary Information accompanies the paper on Cell Death and Differentiation website (http://www.nature.com/cdd)}

